Automorphisms of finite $p$-groups admitting a partition

Khukhro, E. I.

2012

MIMS EPrint: 2012.94

Manchester Institute for Mathematical Sciences

School of Mathematics

The University of Manchester

\footnotetext{
Reports available from: http://eprints.maths.manchester.ac.uk/

And by contacting: The MIMS Secretary

School of Mathematics

The University of Manchester

Manchester, M13 9PL, UK
} 


\title{
Automorphisms of finite $p$-groups admitting a partition
}

\author{
E. I. Khukhro
}

\begin{abstract}
For a finite $p$-group $P$ the following three conditions are equivalent: (a) to have a (proper) partition, that is, to be the union of some proper subgroups with trivial pairwise intersections; (b) to have a proper subgroup outside of which all elements have order $p$; (c) to be a semidirect product $P=P_{1} \rtimes\langle\varphi\rangle$ where $P_{1}$ is a subgroup of index $p$ and $\varphi$ is a splitting automorphism of order $p$ of $P_{1}$. It is proved that if a finite $p$-group $P$ with a partition admits a soluble group of automorphisms $A$ of coprime order such that the fixed-point subgroup $C_{P}(A)$ is soluble of derived length $d$, then $P$ has a maximal subgroup that is nilpotent of class bounded in terms of $p, d$, and $|A|$. The proof is based on a similar result of the author and Shumyatsky for the case where $P$ has exponent $p$ and on the method of "elimination of automorphisms by nilpotency", which was earlier developed by the author, in particular, for studying finite $p$-groups with a partition. It is also proved that if a finite $p$-group $P$ with a partition admits a group of automorphisms $A$ that acts faithfully on $P / H_{p}(P)$, then the exponent of $P$ is bounded in terms of the exponent of $C_{P}(A)$. The proof of this result is based on the author's positive solution of the analogue of Restricted Burnside Problem for finite $p$-groups with a splitting automorphism of order $p$. Both theorems yield corollaries on finite groups admitting a Frobenius group of automorphisms whose kernel is generated by a splitting automorphism of prime order.
\end{abstract}

\section{Introduction}

A finite group $G$ is said to have a (proper) partition if it is the union of some proper subgroups with trivial pairwise intersections: $G=\bigcup_{i} G_{i}$ with $G_{j} \cap G_{k}=1$. The theory of such groups reflects many aspects of the theory of finite groups; for example, nonsoluble finite groups with this property were characterized by Zassenhaus and Suzuki as an important part of the classification program. The monograph [1] contains a description of finite groups with a partition "modulo finite $p$-groups".

For a finite $p$-group $P$ the following three conditions are equivalent:

(a) to have a (proper) partition;

(b) to have a proper subgroup outside of which all elements have order $p$; this obviously means that $P$ has proper Hughes subgroup: $H_{p}(P)=\left\langle g \in P \mid g^{p} \neq 1\right\rangle \neq P$; 
(c) to be a semidirect product $P=P_{1} \rtimes\langle\varphi\rangle$, where $P_{1}$ is a subgroup of index $p$ and $\varphi$ is a splitting automorphism of order $p$ of $P_{1}$, that is,

$$
\varphi^{p}=1 \quad \text { and } \quad x x^{\varphi} x^{\varphi^{2}} \cdots x^{\varphi^{p-1}}=1 \quad \text { for all } x \in P_{1}
$$

Note that in (1) in condition (c) we deliberately do not exclude the case where $\varphi$ acts trivially on $P_{1}$, when, of course, $P_{1}$ must be a group of exponent $p$. Experience shows that in many respects the approach related to splitting automorphisms (condition (c)) is most efficient in the study of finite $p$-groups with a partition. In particular, note that all groups admitting a splitting automorphism of order $p$ constitute a variety of groups with operators defined by the laws (1).

Finite $p$-groups $P$ with a partition can be regarded as close to groups of exponent $p$ : either because all elements outside a proper subgroup are of order $p$, or because the operator law $x x^{\varphi} x^{\varphi^{2}} \cdots x^{\varphi^{p-1}}=1$ turns into the law $x^{p}=1$ when $\varphi=1$. (Note, however, that there is no bound for the exponent of a $p$-group with a partition.) Many properties of groups of exponent $p$ have been extended to finite $p$-groups with a partition. For example, in [2] it was proved that if the subgroup $P_{1}$ in condition (c) is soluble of derived length $d$, then $P_{1}$ is nilpotent of class bounded in terms of $p$ and $d$ (for short, $(p, d)$-bounded), which is an analogue of the corresponding result for groups of exponent $p$. Furthermore, based on the celebrated theorem of Kostrikin for groups of exponent $p$, an analogue of the affirmative solution of the Restricted Burnside Problem was obtained for such groups: it was proved in [3] that the nilpotency class of $P_{1}$ is bounded in terms of $p$ and the number of generators. These and other results can be found in [4, Ch. 6, 7].

In this note we extend to finite $p$-groups with a partition the following result on groups of prime exponent $p$ due to the author and Shumyatsky [5]: if a finite group $G$ of exponent $p$ admits a soluble group of automorphisms $A$ of coprime order such that the fixed-point subgroup $C_{G}(A)$ is soluble of derived length $d$, then $G$ is nilpotent of $(p, d,|A|)$-bounded class.

Theorem 1. Suppose that a finite p-group $P$ with a partition admits a soluble group of automorphisms $A$ of coprime order such that the fixed-point subgroup $C_{P}(A)$ is soluble of derived length $d$. Then any maximal subgroup of $P$ containing $H_{p}(P)$ is nilpotent of class bounded in terms of $p, d$, and $|A|$.

Examples show that the nilpotency class of the whole group $P$ cannot be bounded. The bound for the nilpotency class of that maximal subgroup can be chosen to be the same as the bound in the aforementioned result of [5] for groups of exponent $p$. The proof is based on the theorem in [5] for groups of exponent $p$ and the method of "elimination of automorphisms by nilpotency", which was developed earlier by the author, in particular, for studying finite $p$-groups with a splitting automorphism of order $p$; see $[6]$ and $[4$, Ch. 6].

Another result concerns bounding the exponent.

Theorem 2. If a finite p-group $P$ with a partition admits a group of automorphisms $A$ that acts faithfully on $P / H_{p}(P)$, then the exponent of $P$ is bounded in terms of the exponent of $C_{P}(A)$. 
The proof of this result is based on the author's positive solution of the analogue of Restricted Burnside Problem for finite $p$-groups with a splitting automorphism of order $p$.

Both theorems yield corollaries for finite groups with certain Frobenius groups of automorphisms.

Corollary. Suppose that a finite group $G$ admits a Frobenius group of automorphisms $F H$ with complement $H$ and with cyclic kernel $F=\langle\varphi\rangle$ of prime order $p$ such that $\varphi$ is a splitting automorphism, that is, $x x^{\varphi} x^{\varphi^{2}} \cdots x^{\varphi^{p-1}}=1$ for all $x \in G$.

(a) If $C_{G}(H)$ is soluble of derived length $d$, then $G$ is nilpotent of $(p, d)$-bounded class.

(b) The exponent of $G$ is bounded in terms of $p$ and the exponent of $C_{G}(H)$.

Proof. The group $G$ is nilpotent by the Kegel-Thompson-Hughes theorem $[7,8]$. The automorphism $\varphi$ acts fixed-point-freely on its Hall $p^{\prime}$-subgroup $G_{p^{\prime}}$ : for any $g \in C_{G}(\varphi)$ we have $1=g g^{\varphi} g^{\varphi^{2}} \cdots g^{\varphi^{p-1}}=g^{p}$. Hence $G_{p^{\prime}}$ is nilpotent of $p$-bounded class by the Higman-Kreknin-Kostrikin theorem $[9,10,11]$. To complete the proof of part (a) it now remains to consider the Sylow $p$-subgroup $G_{p}$ of $G$. The result for $G_{p}$ follow from Theorem 1 applied to $P=G_{p}\langle\varphi\rangle$ and $A=H$.

By Lemma 2.4 in [12] (see also [13, Lemma 2]) we have $G_{p^{\prime}}=\left\langle C_{G_{p^{\prime}}}(H)^{f} \mid f \in F\right\rangle$. This implies that $G_{p^{\prime}}$ is generated by elements of orders dividing the exponent of $C_{G}(H)$. Together with the bound in terms of $p$ for the nilpotency class of $G_{p^{\prime}}$, this gives a bound for the exponent of $G_{p^{\prime}}$ in terms of $p$ and the exponent of $C_{G}(H)$. Thus, in part (b) it remains to consider the Sylow $p$-subgroup $G_{p}$ of $G$. The result for $G_{p}$ follow from Theorem 2 applied to $P=G_{p}\langle\varphi\rangle$ and $A=H$.

Examples show that one cannot get rid of the dependence of the nilpotency class on $p$ in part (a) of the corollary (which is obviously also true for the exponent in part (b)). Note that there are other recent papers $[12,13,14,15,16,17,18]$ on finite groups $G$ with a Frobenius group of automorphisms $F H$ with fixed-point-free kernel $F$. In particular, V.D. Mazurov's problem 17.72(a) from Kourovka Notebook [19] on double Frobenius groups was solved in the affirmative in [15], and then in [12], for any metacyclic Frobenius groups of automorphisms $F H$, a bound for the nilpotency class of $G$ was obtained in terms of $|H|$ and the nilpotency class of $C_{G}(H)$, as well as a bound for the exponent of $G$ in terms of $|F H|$ and the exponent of $C_{G}(H)$. The question arises whether similar results, of the type of the above corollary, can be obtained for Frobenius groups of automorphisms with kernel generated by a splitting automorphism of composite order. It is easy to produce examples of finite $p$-groups of unbounded nilpotency class admitting a Frobenius group of automorphisms with cyclic kernel of order $p^{2}$ generated by a splitting automorphism and with complement of order 2 having abelian fixed-point subgroup. But the question remains open on bounding the exponent in such situations with kernel of composite order.

\section{Preliminaries}

The induced group of automorphisms of an invariant section is often denoted by the same letter. Both the group and a group of its automorphisms are regarded as subgroups of 
their semidirect product, so the fixed-point subgroup is denoted as the centralizer, and commutator subgroups can be formed. The actions of homomorphisms and automorphisms are denoted by exponent notation: $a^{\vartheta}$ is the image of $a$ under $\vartheta$. A subgroup $H$ is said to be invariant under an endomorphism $\vartheta$ if $H^{\vartheta} \leqslant H$.

We use the standard notation for simple (left-normed) commutators: $\left[x_{1}, x_{2}, \ldots, x_{k}\right]=$ $\left[\ldots\left[\left[x_{1}, x_{2}\right], x_{3}\right], \ldots, x_{k}\right]$.

Throughout the paper we shall need the following well-known property of coprime action.

Lemma 1. If $A$ is group of automorphisms of a finite group $G$ of coprime order, $(|G|,|A|)=$ 1 , then $C_{G / N}(A)=C_{G}(A) N / N$ for any $A$-invariant normal subgroup $N$.

In other words, $C_{G^{\vartheta}}(A)=\left(C_{G}(A)\right)^{\vartheta}$ for any homomorphism $\vartheta$ of $G$ with $A$-invariant kernel.

We state for convenience of the reader two main results of [5]. Recall that a quantity is said to be $(a, b, \ldots)$-bounded if it is bounded above by a function depending only on $a, b, \ldots$

Theorem 3 ([5, Theorem 4]). If a finite group $G$ of prime exponent $p$ admits a soluble group of automorphisms $A$ of coprime order such that the fixed-point subgroup $C_{G}(A)$ is soluble of derived length $d$, then $G$ is nilpotent of $(p, d,|A|)$-bounded class.

This theorem was proved on the basis of a similar result for $(p-1)$-Engel Lie algebras, which we also need in this paper. Recall that a Lie algebra $L$ is said to be $(p-1)$-Engel if $[x, \underbrace{y, \ldots, y}_{p-1}]=0$ for all $x, y \in L$.

Theorem 4 ([5, Theorem 3]). If a $(p-1)$-Engel Lie algebra L of characteristic $p($ or 0$)$ admits a finite soluble group of automorphisms $A$ of order coprime to the characteristic such that the fixed point subalgebra $C_{L}(A)$ is soluble of derived length $d$, then the Lie algebra $L$ is nilpotent of $(p, d,|A|)$-bounded class.

In the main cases in the proofs of both Theorems 1 and 2 it is required that the group of automorphisms $A$ normalizes a cyclic subgroup $\langle\varphi\rangle \leqslant P$ outside the Hughes subgroup $H_{p}(P)$ and the semidirect product $\langle\varphi\rangle \rtimes A$ acts on a maximal $A$-invariant subgroup of $P$ in such a way that $\varphi$ is a splitting automorphism. If $\left|P: H_{p}(P)\right|=p$, then the maximal subgroup in question is simply $H_{p}(P)$ and an element $\varphi$ can be found without difficulty, by an analogue of Maschke's theorem, see Lemma 2 below. Fortunately, in the situation where $\left|P: H_{p}(P)\right| \geqslant p^{2}$ there is a lot more information about the group $P$ : then $P$ is even closer to being of exponent $p$, in a certain precise sense, and both theorems are easily derived from known results.

Reduction to the case $\left|\boldsymbol{P}: \boldsymbol{H}_{\boldsymbol{p}}(\boldsymbol{P})\right|=\boldsymbol{p}$ in Theorem 1. Recall that the associated Lie ring $L(P)$ is defined on the direct sum of additively written factors of the lower central series of $P$

$$
L(P)=\bigoplus_{i} \gamma_{i} / \gamma_{i+1},
$$


with Lie products defined for the summands via group commutators as

$$
\left[a+\gamma_{i+1}, b+\gamma_{j+1}\right]=[a, b]+\gamma_{i+j+1} \quad \text { for } a \in \gamma_{i}, b \in \gamma_{j}
$$

and extended to $L(P)$ by linearity. It is important that the nilpotency class of $L(P)$ is equal to the nilpotency class of $P$.

Suppose that $P$ is a finite $p$-group with $\left|P: H_{p}(P)\right| \geqslant p^{2}$. It was proved in [20] (see also [4, Theorem 7.3.7]) that then the associated Lie ring $L(P)$ is a $(p-1)$-Engel Lie algebra of characteristic $p$. Suppose that in addition $P$ satisfies the hypotheses of Theorem 1. Consider the induced group of automorphisms $A$ of $L=L(P)$. By Lemma 1 , the fixed-point subalgebra $C_{L}(A)$ is the sum of the images of $C_{P}(A)$ in the factors of the lower central series due to the coprimeness of the action. Therefore it is easy to show that $C_{L}(A)$ is soluble of derived length not higher than the derived length $d$ of $C_{P}(A)$.

By applying Theorem 4 to $L$ we obtain that $L$ is nilpotent of $(p, d,|A|)$-bounded class. Hence $P$ is also nilpotent of the same nilpotency class, and Theorem 1 is proved in the case $\left|P: H_{p}(P)\right| \geqslant p^{2}$.

Reduction to the case $\left|\boldsymbol{P}: \boldsymbol{H}_{p}(\boldsymbol{P})\right|=\boldsymbol{p}$ in Theorem 2. Furthermore, it was proved in [21] (see also [4, Corollary 7.3.8]) that if $\left|P: H_{p}(P)\right| \geqslant p^{2}$, then the exponent of $P$ is bounded in terms of $p$ alone.

Thus, in both Theorems 1 and 2 it remains to consider the main cases where $\mid P$ : $H_{p}(P) \mid=p$, to which the next sections are devoted.

Maschke type lemma. Of course, $H_{p}(P)$ is $A$-invariant, being a characteristic subgroup. The following lemma produces an $A$-invariant complement of $H_{p}(P)$; it is more convenient to prove it in a greater generality.

Lemma 2. Suppose that $A$ is $p^{\prime}$-group of automorphisms of a finite p-group $P$ and $P$ has an $A$-invariant subgroup $P_{1}$ of index $p$. Then there is an A-invariant cyclic subgroup $\langle f\rangle$ such that $P=P_{1}\langle f\rangle$.

Proof. Induction on $|P|$, with obvious basis when $|P|=p$. In the general case, consider the Frattini quotient $P / \Phi(P)$ as an $\mathbb{F}_{p} A$-module, in which the image of $P_{1}$ is an $A$ invariant subspace of codimension 1. By Maschke's theorem the image of $P_{1}$ has an $A$-invariant complement. Choose an element $f_{1}$ in the inverse image of a generator of this complement; then $\left\langle f_{1}\right\rangle P_{1}=P$. In the $A$-invariant subgroup $\left\langle f_{1}\right\rangle \Phi(P)$, the subgroup $\Phi(P)$ is $A$-invariant and has index $p$. By induction, there is an $A$-invariant cyclic subgroup $\langle f\rangle$ such that $\langle f\rangle \Phi(P)=\left\langle f_{1}\right\rangle \Phi(P)$. Then also $\langle f\rangle \Phi(P) P_{1}=\left\langle f_{1}\right\rangle \Phi(P) P_{1}=P$, whence $\langle f\rangle P_{1}=P$.

\section{Bounding the nilpotency class}

In the previous section the proof of Theorem 1 was reduced to the case where $\mid P$ : $H_{p}(P) \mid=p$. By Lemma 2 and due to the fact any element outside $H_{p}(P)$ induces by conjugation a splitting automorphism of order $p$ of $H_{p}(P)$, the proof of Theorem 1 is 
reduced to the following result (which is stated in a slightly greater generality than the case where $\left.\left|P: H_{p}(P)\right|=p\right)$.

Theorem 5. Suppose that a soluble group $F A$ with normal Sylow p-subgroup $F=\langle\varphi\rangle$ of order $p$ and Hall p'-subgroup $A$ acts by automorpisms on a finite p-group $G$ in such a manner that $\varphi$ is a splitting automorphism, that is, $x x^{\varphi} x^{\varphi^{2}} \cdots x^{\varphi^{p-1}}=1$ for all $x \in G$. If $C_{G}(A)$ is soluble of derived length $d$, then $G$ is nilpotent of $(p, d,|A|)$-bounded class.

Proof. The proof is based on the method of "elimination of automorphisms by nilpotency" developed by by the author, which was used, in particular, for studying splitting automorphisms of prime order [2], [4, Ch. 6]. If $\varphi$ acts trivially on $G$, then the group $G$ has exponent $p$ and then the theorem reduces to Theorem 3 ; let $c=c(p, d,|A|)$ be the bound for the nilpotency class given by Theorem 3 in this case. We are actually going to prove that the nilpotency class of $G$ in the general case is also bounded by the same number $c$. Thus, we need to prove that $\left[g_{1}, \ldots, g_{c+1}\right]=1$ for any $g_{i} \in G$. We can assume that $G=\left\langle\left\{g_{1}, \ldots, g_{c+1}\right\}^{F A}\right\rangle$, that is, $G$ is generated by the elements $g_{1}, \ldots, g_{c+1}$ as an $F A$-group.

Henceforth, $F A$-groups are groups with operators $F A$, that is, groups with additional unary operations corresponding to the action of elements of the group $F A$ by automorphisms. By $F A$-homomorphisms we mean homomorphisms in the sense of $F A$-groups, that is, homomorphisms commuting with the action of $F A$; similarly, by (normal) subgroups we mean $F A$-invariant (normal) subgroups. We shall use the word "abstract(ly)" to specify (normal) subgroups or other objects in the abstract sense, irrespective of an action of $F A$.

Let $p^{m}$ be the exponent of $G$, and $n$ the nilpotency class of $G$. (It is of course important that the bound for the nilpotency class of $P$ that we shall obtain will be independent of these parameters.) Consider a (relatively) free $F A$-group $X$ on free generators $x_{1} \ldots, x_{c+1}$ in the variety of nilpotent $F A$-groups of class $n$ and of exponent $p^{m}$. This means that $X$ is an abstract group that is free in the variety of nilpotent groups of class $n$ and of exponent $p^{m}$ on the free generators $x_{i}^{y}$, where $y \in F A$, which $F A$ permutes naturally: $\left(x_{i}^{h}\right)^{z}=x_{i}^{y z}$ for $y, z \in F A$. (Here the elements $x_{i}$ are identified with the elements $x_{i}^{e}$, where $e$ is the identity element of $F A$.)

Note that $X$ is a finite $p$-group, being a finitely generated nilpotent group of exponent $p^{m}$. Hence the semidirect product $X F$ is also a finite $p$-group and is therefore nilpotent of some class.

There is an $F A$-homomorphism $\xi: X \rightarrow G$ extending the mapping $x_{i} \rightarrow g_{i}, i=$ $1, \ldots, c+1$. We simply need to show that $\left[x_{1}, \ldots, x_{c+1}\right] \in \operatorname{Ker} \xi$. Note that $\operatorname{Ker} \xi$ is an $F A$-invariant normal subgroup of $X$.

Let $C=\left\langle\left(\left(C_{X}(A)\right)^{(d)}\right)^{X F A}\right\rangle$ be the $F A$-invariant normal closure of the $d$ th term $\left(C_{X}(A)\right)^{(d)}$ of the derived series of $C_{X}(A)$. Since $X$ is a finite $p$-group, while $A$ is a $p^{\prime}$ group, we have $C_{G}(A)=\left(C_{X}(A)\right)^{\xi}$ by Lemma 1 . By hypothesis, $\left(C_{G}(A)\right)^{(d)}=1$; hence, $\left(C_{X}(A)\right)^{(d)} \leqslant \operatorname{Ker} \xi$ and therefore, $C \leqslant \operatorname{Ker} \xi$. Note also that $C_{X / C}(A)$ is soluble of derived length $d$, being the image of $C_{X}(A)$ by Lemma 1 .

Let

$$
S=\left\langle\left\{x x^{\varphi} x^{\varphi^{2}} \cdots x^{\varphi^{p-1}} \mid x \in X\right\}^{X F A}\right\rangle
$$


be the $F A$-invariant normal closure of the set of all the products indicated in braces. Since all these products are trivial in $G$ by hypothesis, we have $S \leqslant \operatorname{Ker} \xi$.

Thus, it is sufficient (and actually even necessary) to prove that $\left[x_{1}, \ldots, x_{c+1}\right] \in C S$.

Lemma 3. The subgroups $C$ and $S$ are invariant under any $F A$-endomorphism $\vartheta$ of $X$.

Proof. Any $F A$-endomorphism $\vartheta: X \rightarrow X$ commutes with the action of $F A$. In particular, $C_{X}(A)$ is $\vartheta$-invariant: for any $x \in C_{X}(A)$ and $a \in A$ we have $\left(x^{\vartheta}\right)^{a}=\left(x^{a}\right)^{\vartheta}=x^{\vartheta}$. Then clearly $C_{X}(A)^{(d)}$ is also $\vartheta$-invariant. Any element in $C$ is a product of elements of the form $z^{x f a}$, where $z \in C_{X}(A)^{(d)}, x \in X, f a \in F A$. The image of such an element under $\vartheta$ is $\left(z^{x f a}\right)^{\vartheta}=\left(\left(z^{x}\right)^{\vartheta}\right)^{f a}=\left(\left(z^{\vartheta}\right)^{x^{\vartheta}}\right)^{f a}$, which again has the same form, since $z^{\vartheta} \in C_{X}(A)^{(d)}$. Hence $C$ is $\vartheta$-invariant.

For $S$, the image under $\vartheta$ of a product of elements of the form

$$
\left(x x^{\varphi} x^{\varphi^{2}} \cdots x^{\varphi^{p-1}}\right)^{y f a}, \quad x, y \in X, f \in F, a \in A,
$$

is again a product of elements of the same form

$$
\left(\left(x x^{\varphi} x^{\varphi^{2}} \cdots x^{\varphi^{p-1}}\right)^{y f a}\right)^{\vartheta}=\left(\left(x^{\vartheta}\right)\left(x^{\vartheta}\right)^{\varphi}\left(x^{\vartheta}\right)^{\varphi^{2}} \cdots\left(x^{\vartheta}\right)^{\varphi^{p-1}}\right)^{y^{\vartheta} f a},
$$

where we used the fact that $\vartheta$ commutes with $F A$.

Remark 1. Lemma 3 essentially says that $C$ and $S$ are verbal $F A$-subgroups of $X$. This fact is fairly obvious for $S$. But this is less obvious for $C$, since the centralizer of a group of automorphisms in general may not be given by group words with operators. Nevertheless, this is true in Lemma 3 due to the coprimeness of the action. An alternative way of proving this lemma would be by using Birkhoff's theorem. For $S$ (or $C$ ), it is sufficient to show that the class of $F A$-groups $G$ in which $x x^{\varphi} x^{\varphi^{2}} \cdots x^{\varphi^{p-1}}=1$ for all $x \in G$ (respectively, $C_{G}(A)^{(d)}=1$ ) is closed under taking $F A$-homomorphic images, $F A$ subgroups, and Cartesian products. Actually, the condition $C_{G}(A)^{(d)}=1$ may not be preserved under $F A$-homomorphic images, but if we restrict ourselves to nilpotent groups of class $n$ and of exponent $p^{m}$, then the coprimeness of the action of $A$ comes to the rescue.

Let $T$ be the (abstract) normal closure of $F$ in $X F$; abstractly, $T=[X, F] F$. Note that both $X$ and $F$ are $F A$-invariant, so $T$ is also $F A$-invariant. Factorization by $T$ amounts to "trivialization" of $\varphi$ : in the quotient $(X F A) /(C S T)$ the image of $\varphi$ becomes trivial. Hence the image of $X$ in $(X F A) /(C S T)$ is of exponent $p$, since this image also satisfies the identity

$$
\bar{x} \bar{x}^{\varphi} \bar{x}^{\varphi^{2}} \cdots \bar{x}^{\varphi^{p-1}}=1, \quad \bar{x} \in(X C S T) /(C S T) .
$$

The centralizer of $A$ in this quotient is the image of the centralizer of $A$ in $X / C$ by Lemma 1 , since the action of $A$ on $X / C$ is coprime. Therefore $C_{(X C S T) /(C S T)}(A)$ is also soluble of derived length $d$. We can now apply Theorem 3 to $(X C S T) /(C S T)$ and $A$ to conclude that $(X C S T) /(C S T)$ is nilpotent of class $c$. This means that

$$
\left[x_{1}, \ldots, x_{c+1}\right] \in C S T \text {. }
$$

All we need is to eliminate $T$ from inclusion (2). 
We rewrite (2) as a congruence modulo $C S$ :

$$
\left[x_{1}, \ldots, x_{c+1}\right] \equiv t(\bmod C S),
$$

where $t \in T$.

As an abstract group, $X F$ is generated by the elements $\varphi$ and $x_{i}^{a_{*}}$, where $a_{*} \in A$ denote various elements of $A$. Since the semidirect product $X F$ is a nilpotent finite $p$-group, we can apply the standard collecting process arguments with respect to these generators. Here we can avoid using any negative powers of commutators, since $a^{-1}=a^{|a|-1}$ for any element $a$ of finite order. This technical remark allows us to somewhat simplify subsequent arguments.

Every element of $T=[X, F] F$ is a product of commutators in these elements, including those of weight 1, each involving at least one occurrence of $\varphi$ (so in fact the only such commutator of weight 1 is $\varphi$ ). Indeed, such products of commutators clearly form an abstract normal subgroup of $X F$ containing $\varphi$, and are contained in $[X, F] F$. (Recall that in a periodic group inverse elements are also obtained in this fashion.) Since $[X, F] F$ is the minimal abstract normal subgroup of $X F$ containing $F$, it must coincide with the set of such products. Thus, we rewrite (3) as

$$
\left[x_{1}, \ldots, x_{c+1}\right] \equiv c_{1} \cdots c_{m}(\bmod C S)
$$

where the $c_{i}$ are commutators in $\varphi$ and $x_{i}^{a_{*}}$ each involving at least one occurrence of $\varphi$.

We say for brevity that a commutator in elements $\varphi$ and $x_{i}^{a_{*}}$ depends on $\varphi$ if it involves $\varphi$ as an entry, and depends on $x_{j}$ (for a given $j$ ) if it involves at least one entry $x_{j}^{a_{*}}$ for some $a_{*} \in A$.

Lemma 4. We can assume that each commutator $c_{s}$ in (4) depends on $\varphi$ and on all the $x_{i}, i=1, \ldots, c+1$.

(In other words, in addition to dependence on $\varphi$, all $i=1, \ldots, c+1$ occur as lower indices of $x_{i}^{a_{*}}$ in each $c_{s}$, where the $a_{*}$ may be the same or different.)

Proof. This is proved similarly to the so-called Higman's lemma, by applying the $F A$ endomorphisms $\vartheta_{i}$ of $X$ extending the mapping $x_{i} \rightarrow 1, x_{j} \rightarrow x_{j}$ for $j \neq i$. As an abstract endomorphism $\vartheta_{i}$ is defined by the mapping $x_{i}^{f a} \rightarrow 1$ for all $f a \in F A$ and $x_{j}^{f a} \rightarrow x_{j}^{f a}$ for $j \neq i$. This is an $F A$-endomorphism of $X$, that is, commuting with the action of $F A$, because the $x_{k}$ are free generators of the (relatively free) $F A$-group $X$.

By Lemma 3, both $S$ and $C$ are $\vartheta_{i}$-invariant. Therefore an application of $\vartheta_{i}$ to a congruence modulo $C S$ produces again a congruence modulo $C S$. For a given $i$, when $\vartheta_{i}$ is applied to (4), the left-hand side goes to 1 , as do all the $c_{s}$ on the right that depend on $x_{i}$. As a result, the product of commutators on the right that do not depend on $x_{i}$ belongs to $C S$ and can be dropped in (4). More accurately, first all the commutators that do not depend on $x_{i}$ are collected (in some order) at the beginning of the product on the right of (4). For that we need to transpose commutators, but this gives rise only to new commutators depending on all the variables on which either of the commutators being transposed depended. So in such a collecting process all the dependencies are preserved, including dependence on $\varphi$. After consecutively eliminating all commutators that do not depend on $x_{i}$ for each $i=1, \ldots, c+1$, we arrive at a congruence (4) in which each of the $c_{s}$ depends on all the $x_{i}$ (and on $\varphi$ ). 
Lemma 5. Every commutator in elements $x_{i}^{a_{*}}$ and $\varphi$ depending on $\varphi$ and all of the $x_{1}, \ldots x_{c+1}$ can be represented as a product of commutators of the form

$$
\left[\left[x_{i_{1}}^{a_{*}}, \ldots\right],\left[x_{i_{2}}^{a_{*}}, \ldots\right], \ldots\left[x_{i_{c+1}}^{a_{*}}, \ldots\right]\right]
$$

which is a simple commutator in simple subcommutators, such that $\left\{i_{1}, i_{2}, \ldots, i_{c+1}\right\}=$ $\{1,2, \ldots, c+1\}$, the dots in each simple subcommutator $\left[x_{i_{s}}^{a_{*}}, \ldots\right]$ denote (possibly absent) elements $\varphi$ or $x_{i}^{a_{*}}$ in any combination and any order, and the total number of occurrences of $\varphi$ in each commutator (5) is at least the number of occurrences of $\varphi$ in the original commutator.

Proof. This follows from the standard commutator formulae

$$
\begin{aligned}
{[u,[v, w]] } & =[[u, v], w] \cdot[[u, w], v]^{-1} \cdot \varkappa \\
{[[u, v], w] } & =[[v, u], w]^{-1} \cdot \varkappa \\
{[[u, v], w]] } & =[[u, w], v] \cdot[u,[v, w]] \cdot \varkappa,
\end{aligned}
$$

which hold in a nilpotent group, where in each case $\varkappa$ is a product of powers of commutators in the same elements $u, v, w$ each involving all these elements $u, v, w$. In our group $X F$, negative powers of commutators here can again be replaced by appropriate positive powers of these commutators, which is always possible in a periodic group, and in what follows we shall apply formulae (6), (7), (8) using such replacements.

In more detail: for example, firstly the commutator is represented as a product of simple commutators by "expanding" all inner brackets by formula (6). This formula is applied to inner brackets one at a time. When additional factors appear, the formula is always applied to one of non-simple commutators of the lowest weight. At every step, apart from "improved" commutators of type $[[u, v], w]$ and $[[u, w], v]$, only commutators of greater weight will appear, and all the new commutators will be depending on the same elements as the commutator that is being transformed, with at least the same multiplicity of occurrence of each element (including $\varphi$ ). At each step, commutators of lower weights, which are already simple commutators, are not affected. The process is finite, since $X F$ is a nilpotent group of finite exponent.

Thus, we can assume from the outset that the commutator is simple. In such a commutator we can locate one of the occurrences of each of the $x_{1}, \ldots x_{c+1}$ :

$$
\left[\ldots, x_{i_{1}}^{a_{*}}, \ldots x_{i_{2}}^{a_{*}}, \ldots \ldots, x_{i_{c+1}}^{a_{*}}, \ldots\right]
$$

where $\left\{i_{1}, i_{2}, \ldots, i_{c+1}\right\}=\{1,2, \ldots, c+1\}$ and the dots denote elements $\varphi$ or $x_{i}^{a_{*}}$ with at least the same multiplicity of occurrence of $\varphi$. Then we can use the formula (7) to put $x_{i_{1}}^{a_{*}}$ at the start. After that we collect all the elements denoted by dots to place them right after the first element $x_{i_{1}}^{a_{*}}$ by moving them by formula (8) to the left one at a time, always moving to the left the left-most of the non-collected elements. The additional factors will of course arise, when the elements are transferred over those fixed occurrences, or rather over the "growing" subcommutators $\left[x_{i_{j}}^{a_{*}}, \ldots\right]$ that emerge in their place. All the additional factors of higher weight will depend on $\varphi$ with at least the same multiplicity of occurrence, and on each of the $x_{1}, \ldots x_{c+1}$. At each step of this process we 
shall obtain a product of commutators of the required form and commutators of greater weight satisfying the original hypothesis of the lemma. The same process, starting from expressing in terms of simple commutators described above, is applied to them, and so on. Since the group $X F$ is nilpotent and has finite exponent, this process will terminate resulting in a required product.

By Lemmas 4 and 5, we can assume that each commutator $c_{i}$ in (4) has the form (5).

We now finish the proof of the theorem by an iterative "self-amplification" of the congruence (4) when its consequences are applied to itself. At each step of this process, assuming that all the $c_{i}$ on the right of (4) are of the form (5), we apply consequences of (4) to express these $c_{i}$ in terms of commutators of the same form but with ever increasing number of occurrences of $\varphi$. Since the group $X F$ is nilpotent, this will eventually mean that the right-hand side of (4) becomes trivial, which proves the theorem.

Let $c_{i}$ be one of the commutators on the right of (4); we assume that $c_{i}$ has the form (5). Consider the $F A$-endomorphism $\vartheta$ of $X$ extending the mapping

$$
x_{j} \rightarrow\left[x_{i_{j}}^{a_{*}}, \ldots\right], \quad j=1, \ldots, c+1,
$$

where $\left[x_{i_{j}}^{a_{*}}, \ldots\right]$ are the subcommutators in $c_{i}$ of the form (5). This mapping can indeed be extended to an $F A$-endomorphism of $X$ because the $x_{i}$ are free generators of the relatively free $F A$-group $X$. By Lemma 3 , both $C$ and $S$ are $\vartheta$-invariant. Therefore, when $\vartheta$ is applied to (4) we obtain again a congruence modulo $C S$. As a result, the commutator $c_{i}$ as the image of the left-hand side of (4) is expressed as the image under $\vartheta$ of the righthand side of (4). Note that the image $c_{j}^{\vartheta}$ of each commutator $c_{j}$ on the right of (4) has at least two occurrences of $\varphi$ - at least one was already there originally, and at least another one appears in the image of one of the $x_{k}$ (all of which occurred in $c_{i}$ ) under the mapping (9), since $c_{i}$ of the form (5) also contains an occurrence of $\varphi$.

After expressing in this way each commutator $c_{i}$ on the right in (4) (of the form (5)), we substitute their expressions into the right-hand side of the original congruence (4). Then the resulting commutators on the right-hand side are expressed by Lemma 5 as a product of commutators of the form (5), but now each of them involves at least two occurrences of $\varphi$.

Then we repeat this procedure bearing in mind that in the new congruence (4) each $c_{i}$ has at least two occurrences of $\varphi$. As a result, for the same reasons, we obtain a new congruence (4) in which each $c_{i}$ has at least four occurrences of $\varphi$. We continue this "auto-amplification" process, doubling the number of occurrences of $\varphi$ at each step.

Since the group $X F$ is nilpotent of some class, being a finite $p$-group, in the end the right-hand side of (4) becomes trivial due to unbounded accumulation of occurrences of $\varphi$.

\section{Bounding the exponent}

Here we prove Theorem 2, which has already been reduced in $\S 2$ to the case where $\left|P: H_{p}(P)\right|=p$. By Lemma 2 and due to the fact any element outside $H_{p}(P)$ induces by conjugation a splitting automorphism of order $p$ of $H_{p}(P)$, the proof of Theorem 2 reduces to the following result. 
Theorem 6. If a finite p-group $G$ admits a Frobenius group of automorphisms $F A$ with kernel $F=\langle\varphi\rangle$ of order $p$ and complement $A$ such that $\varphi$ is a splitting automorphism, that is, $x x^{\varphi} x^{\varphi^{2}} \cdots x^{\varphi^{p-1}}=1$ for all $x \in G$, then the exponent of $P$ is bounded in terms of the exponent of $C_{P}(A)$.

Proof. Since any element $g \in G$ belongs to the $F A$-invariant subgroup $\left\langle g^{F A}\right\rangle$, we can obviously assume that $G$ is (abstractly) generated by $|F A|$ elements. By the author's theorem [3] giving an affirmative solution to an analogue of the Restricted Burnside Problem for groups admitting a splitting automorphism of prime order $p$, the nilpotency class of $G$ is bounded in terms of $p$ and the number of generators, which is at most $p(p-1)$. Since the exponents of the factors of the lower central series divide the exponent of the derived quotient group, it remains to obtain a bound for the exponent of $V=$ $G /[G, G]$. We consider this abelian group as a $\mathbb{Z} F A$-module, with additive notation for the group operations and right operator notation for the action of $F A$. In particular, $v+v \varphi+v \varphi^{2}+\cdots+v \varphi^{p-1}=0$ for all $v \in V$ by hypothesis.

We extend the ground ring by a primitive $p$ th root of unity $\omega$, forming $W=V \otimes_{\mathbb{Z}} \mathbb{Z}[\omega]$. We obviously still have the identity $w+w \varphi+w \varphi^{2}+\cdots+w \varphi^{p-1}=0$ for all $w \in W$. We introduce the analogues of eigenspaces for the "linear transformation" $\varphi$, the additive subgroups

$$
W_{i}=\left\{w \in W \mid w \varphi=\omega^{i} w\right\} .
$$

As is well known (see, for example, [4, Lemma 4.1.1]), the $\mathbb{Z}[\omega]\langle\varphi\rangle$-module $W$ is an "almost direct sum" of the $W_{i}$ : namely,

$$
p W \subseteq W_{0}+W_{1}+\cdots+W_{p-1}
$$

and

$$
\text { if } w_{0}+w_{1}+\cdots+w_{p-1}=0 \quad \text { for } \quad w_{i} \in W_{i}, \quad \text { then } \quad p w_{i}=0 \text { for all } i .
$$

Since $\varphi$ acts trivially on $W_{0}$, for $x \in W_{0}$ we have $p x=x+x \varphi+\cdots+x \varphi^{p-1}=0$, so that $p W_{0}=0$.

We now consider the action of $A$. The group $A$ acts on the set of the additive subgroups $W_{i}$ in the same way as $A$ acts on $\langle\varphi\rangle$. Namely, since $F$ is cyclic of order $p$, the group $A$ is also cyclic. Let $A=\langle\alpha\rangle$ and let $\varphi^{\alpha^{-1}}=\varphi^{r}$ for some $1 \leqslant r \leqslant p-1$. Let $|\alpha|=n$; then $r$ is a primitive $n$th root of 1 in $\mathbb{Z} / p \mathbb{Z}$. Then the group $A$ "almost permutes" the additive subgroups $W_{i}$ in the following sense: $W_{i} \alpha \subseteq W_{r i}$ for all $i \in \mathbb{Z} / p \mathbb{Z}$. Indeed, if $x_{i} \in W_{i}$, then $\left(x_{i} \alpha\right) \varphi=x_{i}\left(\alpha \varphi \alpha^{-1} \alpha\right)=\left(x_{i} \varphi^{r}\right) \alpha=\omega^{i r} x_{i} \alpha$.

Given $u_{k} \in W_{k}$ for $k \neq 0$, to lighten the notation we denote $u_{k} \alpha^{i}$ by $u_{r^{i} k}$; note that $u_{r^{i} k} \in W_{r^{i} k}$.

Let $p^{e}$ be the exponent of $C_{G}(A)$. We claim all the $W_{i}$ are annihilated by $p^{1+e}$. For any $k \neq 0$ and for any $u_{k} \in W_{k}$ the sum

$$
u_{k}+u_{k} \alpha+\cdots+u_{k} \alpha^{n-1}=u_{k}+u_{r k}+\cdots+u_{r^{n-1} k}
$$

over the $A$-orbit of $u_{k}$ is a fixed point for $A$. Since the fixed-point submodule $C_{V}(A)$ is annihilated by $p^{e}$ (as the image of $C_{G}(A)$ by Lemma 1 due to the coprimeness of 
the action), the fixed-point submodule $C_{W}(A)$ is also annihilated by $p^{e}$ as it is exactly $C_{V}(A) \otimes_{\mathbb{Z}} \mathbb{Z}[\omega]$. Therefore $p^{e}$ annihilates this sum:

$$
p^{e} u_{k}+p^{e} u_{r k}+\cdots+p^{e} u_{r^{n-1} k}=0 .
$$

We have $u_{r^{i} k} \in W_{r^{i} k}$ and all these indices $k, r k, \ldots, r^{n-1} k$ are distinct modulo $p$. Therefore by (11) the equation (12) implies that, in particular, $p p^{e} u_{k}=0$.

Thus, $p^{1+e} W_{k}=0$ for all $k$ (recall that $p W_{0}=0$ ). As a result, using (10) we obtain

$$
p^{2+e} W \subseteq p^{1+e}\left(W_{0}+W_{1}+\cdots+W_{p-1}\right)=0 .
$$

This also implies that $p^{2+e} V=0$.

Returning to multiplicative notation we obtain that the exponent of $G /[G, G]$ is at most $p^{2+e}$, so the exponent of $G$ is at most $p^{c(2+e)}$, where $c$ is the nilpotency class of $G$, which is bounded in terms of $p$.

Remark 2. If, for some reason, it is known that the derived length $s$ of the group $G$ in Theorem 2 is a relatively small, then we can use the result of [2] (see also [4, Corollary 6.4.2]) to give a possibly smaller estimate $\left((p-1)^{s}-1\right) /(p-2)$ for the nilpotency class of $G$, instead of the one obtained in the proof above, which stems from the estimate in [3], which in turn relies on the estimate for the nilpotency class of a $p(p-1)$-generated $(p-1)$-Engel Lie algebra of characteristic $p$ in Kostrikin's theorem. Correspondingly, there may be a similar improvement in the Corollary, where the nilpotency class may also be given a possibly smaller estimate $\left((p-1)^{s}-1\right) /(p-2)$, since the same number also estimates the nilpotency class of a group admitting a fixed-point-free automorphism of prime order $p$ by the Kreknin-Kostrikin theorem [10]. A smaller bound for the nilpotency class also implies a smaller bound for the exponent. A relatively small derived length would also imply stronger bounds in Theorems 3 and 4, and therefore also in Theorem 1 .

\section{References}

[1] V. M. Busarkin, Yu. M. Gorchakov, Finite splittable groups, Nauka, Moscow, 1968. (Russian)

[2] E. I. Khukhro, Nilpotency of solvable groups admitting a splitting automorphism of prime order, Algebra Logika, 19 (1980), 118-129; English transl., Algebra Logic, 19 (1981), 77-84.

[3] E. I. Khukhro, On locally nilpotent groups admitting a splitting automorphism of prime order, Mat. Sb., 130, no. 1 (1986), 120-127; English transl., Math. USSR-Sb., 58 (1987), 119-126.

[4] E. I. Khukhro, Nilpotent groups and their automorphisms, de Gruyter, Berlin, 1993.

[5] E. I. Khukhro, P. Shumyatsky, On fixed points of automorphisms of Lie rings and locally finite groups, Algebra Logika, 34 (1995), 706-723; English transl., Algebra Logic, 34 (1995), 395-405. 
[6] E. I. Khukhro, Nilpotency in varieties of groups with operators, Mat. Zametki, 50, no. 2 (1991), 142-145 (Russian); English transl., Math. Notes, 50, no. 2 (1991), 869-871.

[7] O. H. Kegel, Die Nilpotenz der $H_{p}$-Gruppen, Math. Z., 75 (1960/1961), 373-376.

[8] D. R. Hughes, J. G. Thompson, The $H_{p}$-problem and the structure of $H_{p}$-groups, Pacific J. Math., 9 (1959), 1097-1101.

[9] G. Higman, Groups and rings which have automorphisms without non-trivial fixed elements, J. London Math. Soc., 32 (1957), 321-334.

[10] V. A. Kreknin, A. I. Kostrikin, Lie algebras with regular automorphisms, Dokl. Akad. Nauk SSSR, 149 (1963), 249-251 (Russian); English transl., Math. USSR Doklady, 4, 355-358.

[11] V.A. Kreknin, The solubility of Lie algebras with regular automorphisms of finite period, Dokl. Akad. Nauk SSSR, 150 (1963), 467-469 (Russian); English transl., Math. USSR Doklady, 4 (1963), 683-685.

[12] E. I. Khukhro, N. Yu. Makarenko, P. Shumyatsky, Frobenius groups of automorphisms and their fixed points, to appear in Forum Math., 2012;

http://arxiv.org/abs/1010.0343.

[13] N. Yu. Makarenko, E. I. Khukhro, P. Shumyatsky, Fixed points of Frobenius groups of automorphisms, Dokl. Akad. Nauk, 437, no. 1 (2011), 20-23; English transl., Dokl. Math., 83, no. 2 (2011), 152-154.

[14] E. I. Khukhro, An application of graded Lie algebras with few components to 2-Frobenius groups, Bull. London Math. Soc., 40 (2008), 907-912.

[15] N. Yu. Makarenko, P. Shumyatsky, Frobenius groups as groups of automorphisms, Proc. Amer. Math. Soc., 138 (2010), 3425-3436.

[16] E. I. Khukhro, Fixed points of the complements of Frobenius groups of automorphisms, Sibirsk. Mat. Zh., 51 (2010), 694-699; English transl., Siberian Math. J., 51 (2010), 554-558.

[17] E. I. Khukhro, Nilpotent length of a finite group admitting a Frobenius group of automorphisms with fixed-point-free kernel, Algebra Logika, 49, no. 6 (2010), 819833; English transl., Algebra Logic, 2010, 49, no. 6 (2011), 551-560.

[18] P. Shumyatsky, On the exponent of a finite group with an automorphism group of order twelve, J. Algebra, 331, no. 1 (2011), 482-489.

[19] Unsolved problems in group theory. The Kourovka Notebook, 17-th ed., Inst. Math. Siber. Div. Russ. Acad. Sci., Novosibirsk, 2010. 
[20] E. I. Khukhro, On finite p-groups that do not satisfy the Hughes conjecture, Sibirsk. Mat. Zh., 35, no. 1 (1994), 221-227; English transl., Siberian Math. J., 35, no. 1 (1994), 202-207.

[21] E. I. Khukhro, On the Hughes problem for finite p-groups, Algebra Logika, 26 (1987), 642-646; English transl., Algebra Logic, 26 (1988), 398-401. 